\title{
miRNA-223 regulates ischemic neuronal injury by targeting the type 1 insulin-like growth factor receptor (IGF1R)
}

\author{
She-Jun Feng ${ }^{1}$, Xue-Qiang Zhang ${ }^{2}$, Jun-Tao $\mathrm{Li}^{1}$, Xiao-Min Dai ${ }^{1}$, Fei Zhao ${ }^{1}$ \\ ${ }^{1}$ Department of Neurology, Handan Central Hospital, ${ }^{2}$ Department of Stomatology, Handan Central Hospital, Handan, China
}

\begin{abstract}
Cerebral ischemia injury seriously endangers human health and its molecular mechanism is still not fully understood. microRNA-223 (miR-223) has been reported to be involved in many physiological functions but the specific role of miRNA-223 in ischemic neuronal injury is still unclear. An oxygen-glucose deprivation and simulated reperfusion $(O G D / R)$ model was constructed here to investigate the possible role miR-223 played in ischemic neuronal injury. The expression of miRNA-223 in the OGD/R model and its effect on cell proliferation were studied by $q P C R$ and CCK8 assay. We observed that miR-223 was significantly over-expressed after OGD/R treatment and it suppressed significantly cortical neurons proliferation. To further study the mechanism involved, we predicted and examined the potential targets of miR-223 by targetscan, GPCR, western blot and luciferase reporter assay. We found that the expression level of type 1 insulin-like growth factor receptor (IGF1R) was negatively associated with the level of miR-223. Furthermore, the relative luciferase activity of pmirGLO-WT was inhibited obviously, while no significant change was observed in the pmirGLO-Mut group, indicating that miR-223 could bind to IGF1R. Similar cell proliferation suppression caused by miR-223 antagomir was observed when IGF1R was silenced. On the contrary, when cortical neurons were co-treated with miR-223 agomir and the CDNA of IGF1R which did not contain 3'- untranslated region, the inhibition caused by miR-223 disappeared. Our results suggested that miR-223 may suppress proliferation of cortical neurons that were treated with OGD/R via inhibiting IGF1R expression.
\end{abstract}

Key words: miR-223, IGF1R, OGD/R, ischemic brain injuries.

\section{Introduction}

Stroke is a common refractory disease that seriously endangers human health and life safety. Majority of all the strokes arise as ischemic brain injuries [17]. Ischemic brain injury is caused by a sudden obstruction of the blood supply, resulting in the reduction of oxygen and glucose supply to the brain tissue [28]. It has a high incidence and high mortality. To date, the molecular mechanism of cere- bral ischemia injury has not been fully understood yet [10]. Moreover, the only effective clinical treatment for ischemic stroke is to dissolve thrombus through intravenous recombinant tissue plasminogen activator [1]. Hence, further explorations for the detailed mechanism of cerebral ischemia injury and treatment strategies are still needed.

MicroRNA (miRNA) is a class of non-coding single-stranded RNA molecules encoded by endoge- 
nous genes of about 20-24 nucleotides and involved in post-transcriptional gene expression regulation [11]. Many studies have demonstrated that a variety of miRNAs are involved in cerebral blood reperfusion injury [3]. Yang et al. [26] have reported that the expression of microRNA-323 was up-regulated after OGD treatment and it might regulate neuronal cell death in an ischemia/reperfusion-induced rat by targeting BRI3. microRNA-134 was found to promote neuron death in hippocampal neurons after OGDinduced ischemic injury [8]. microRNA-223 (miR-223) has attracted a wide range of attention because it is involved in a variety of physiological functions, including cell cycle regulation, immune cell function, hematopoietic differentiation and tumor invasiveness [6]. miR-223 mainly exists in bone marrow and is also highly present in the brain [22]. It has been reported that miR-223 was up-regulated in recurrent ovarian cancer [12] and bladder cancer [5]. Although Wang et al. have reported that miR-223 is up-regulated in acute ischemic stroke patients [22], the specific effects and mechanism of miR-223 in cerebral ischemic injury are still obscure.

The type 1 insulin-like growth factor receptor (IGF1R) is a transmembrane receptor tyrosine kinase, which is involved in regulating the biological activity of IGF. It has been demonstrated that the activation of IGF can inhibit cell apoptosis induced by hypoxia, osmotic stress and anti-cancer drugs [18]. Jia et al. have demonstrated that IGF-1R was the target of miR-223 in HeLa, leukemia and hepatoma cells, and is associated with cell proliferation [9]. However, no significant correlation between miR-223 and IGF1R was found in the blood of acute ischemia patients [22]. Hence, the relationship between miR-223 and IGF1R was still controversial and may be different depending on the types of cells or tissues.

In this paper, we explored the expression and effect of miR-223 in cortical neurons exposed to ischemic insults and investigated the potential mechanism of miR-223-mediated inhibition of cell proliferation. We found that miR-223 may participate in OGD/R by regulating the expression of IGF1R.

\section{Material and methods \\ Primary cortical neuron culture}

The primary cultures of cortical neurons were obtained from the brains of embryonic day 18 (E18) Sprague-Dawley rats as described previously [19].
Cerebral cortices were isolated followed by trypsin digestion. Subsequently, cells were seeded into 24-well plate $\left(1.5 \times 10^{5}\right.$ cells/well $)$ and cultured in the neurobasal medium containing B27 ( $2 \%$, Gibco, USA), glutamine ( $2 \mathrm{mM})$, and $1 \%$ penicillin/streptomycin at $37^{\circ} \mathrm{C}$ with $5 \% \mathrm{CO}_{2}$.

\section{Oxygen-glucose deprivation and simulated reperfusion (OGD/R) model}

The OGD/R model was constructed as described previously with small modifications [15,25]. Briefly, the primary cortical neurons were incubated in an Earle's balanced salt solution (BSS) without glucose at $37^{\circ} \mathrm{C}$ in an anaerobic chamber with $95 \% \mathrm{~N}_{2}$ and $5 \% \mathrm{CO}_{2}$ for $2 \mathrm{~h}$ OGD treatment. Subsequently, the cells were transferred to a normal neurobasal medium and recovered at $37^{\circ} \mathrm{C}$ under a humidified atmosphere of $5 \% \mathrm{CO}_{2}$ and $95 \%$ air. The same treatment was performed in the sham group without OGD exposure.

\section{q-PCR}

Total RNA was extracted from the cortical neurons treated with OGD using Ultrapure RNA kit (CwBio, Beijing, China). cDNA was synthesized using HiFiScript cDNA Synthesis Kit (CwBio, Beijing, China). Fluorescence quantitative PCR was performed to quantify the expressions of miR-223 and IGF1R. The expression levels were calculated by $2-\wedge \wedge C$ method. $\beta$-actin was used as the internal reference gene.

\section{CCK8 assay}

Cell counting kit-8 (CCK-8) (Solarbio, Beijing, China) was used to perform the cell viability assay. The cells in the 96-well cell culture cluster were divided into six groups: sham group, OGD/R group, $\mathrm{OGD} / \mathrm{R}+$ antagomir control group, OGD/R+ antagomir group, OGD/R+ agomir control group and OGD/ $\mathrm{R}+$ agomir group. The final concentration of agomir/ antagomir (Ribobio Co. Ltd., Guangzhou, China) was $5 \mu \mathrm{M}$. Each group contained 5 replicates. In addition to the control group, all the other 5 groups were subjected to OGD/R damage. CCK8 chemical was added to each well and incubating at $37^{\circ} \mathrm{C}$ for $1.5 \mathrm{~h}$, followed by detecting the optical density (OD) at $450 \mathrm{~nm}$ with a microplate reader. 


\section{Transfection}

Cells were grown in six-well plates $\left(1.5 \times 10^{5}\right.$ cells/ well) and maintained overnight before transfection. Then the cortical neurons were transfected with siRNA1 against IGF1R, siRNA2 against IGF1R, siRNA control, vector and pcDNA-TGF1R using Lipofectamine 2000 (Invitrogen, Carlsbad, CA, USA) following the manufacturer's description and cultured for $12 \mathrm{~h}$. Subsequently, cells were treated with OGD/R and harvested for subsequent experiments.

\section{Western blot}

Cells were lysed by RIPA buffer containing protease inhibitor to extract the proteins. The protein concentration was measured with BCA Protein Assay Kit (Com Win Biotech Co., Ltd., Beijing, China). The proteins in the lysates were separated by $12 \%(\mathrm{w} / \mathrm{v})$ SDS-PAGE and then transferred to a polyvinylidene fluoride (PVDF) membrane (Millipore, Bedford, MA). After being blocked in 5\% skimmed milk for $1 \mathrm{~h}$ at room temperature, the membrane was then incubated with TBST containing the primary antibody (1 : 1,000) overnight at $4^{\circ} \mathrm{C}$. Followed by washing with TBST for 3 times, the membrane was next incubated with the secondary antibody $(1: 5,000)$ for $1 \mathrm{~h}$ and washed with TBST for 3 times ( 5 minutes each) again. The signals were detected with an enhanced chemiluminescence detection kit (ECL, Pierce Biotechnology Inc.) and the density was analyzed with Quantity One software. $\beta$-actin was used as an intrinsic quality control. The relative expression level is the ratio of the target protein to the internal reference protein.

\section{Luciferase reporter assay}

The binding site of miR-223 was predicted using TargetScan (http://www.targetscan.org ). The wild type 3'UTR sequence of IGF1R and a mutant with mutations in the predicted binding sites were all synthesized and ligated into the pmirGLO DualLuciferase miRNA Target Expression Vector (Promega, Madison, WI, USA) and named as pmirGLO-WT and pmirGLO-Mut, respectively. Then the cells were transfected with pmirGLO-WT + miR223-agomir control, pmirGLO-WT + miR223-agomir, pmirGLO-Mut + miR223-agomir control or pmirGLO-Mut + miR223-agomir by Lipofectamine 200 (Invitrogen). After transfecting for $48 \mathrm{~h}$, Dual Luciferase Assay
(Promega) was used to test the activities of Firefly and Renilla luciferase consecutively.

\section{Statistical analysis}

The values are shown as mean \pm SD. Student's t-test was performed for single comparison and ANOVA was performed for multiple comparisons. It was considered as statistically significant when $p<0.05$.

\section{Results}

\section{Up-regulated expression of miR-223 after OGD/R}

Primary cortical neurons OGD/R model was used as an in vitro model to explore the role of miR223 in the regulation of ischemic brain damage. According to the results of qPCR (Fig. 1A), we found that the expression level of miR-223 was more significantly elevated in the OGD/R group than in the sham group $(p<0.01)$. To confirm whether miR223 was associated with cortical neurons cell viability after being exposed to OGD, miR223 antagomir and agomir were used and CCK8 assay was performed. As observed in Figure 2B, the level of miR223 was inhibited significantly in the antagomir group and increased obviously in the agomir group, as compared with their corresponding control groups $(p<0.01)$. Cell viability was decreased significantly after OGD/R treatment $(p<0.01)$. When antagomir was added to the cells treated with $\mathrm{OGD} / \mathrm{R}$, the viability was increased more obviously than that of the OGD/R+ antagomir control group $(p<0.01)$. On the other hand, the viability was declined more significantly in the OGD/R+ agomir group than that of the OGD/R+ agomir control group. These results indicated that miR223 may inhibit the proliferation of neurons after OGD and then lead to the ischemic brain damage.

\section{The influence of miR-223 on IGF1R expression}

In order to further explore the mechanism of miR223 inhibition of the proliferation of neurons after OGD, we analyzed its potential targets using the targetscan algorithm. By screening the genes which were down-regulated by miR223 and had been reported to be associated with cancers, IGF1R was selected for further study. We found 

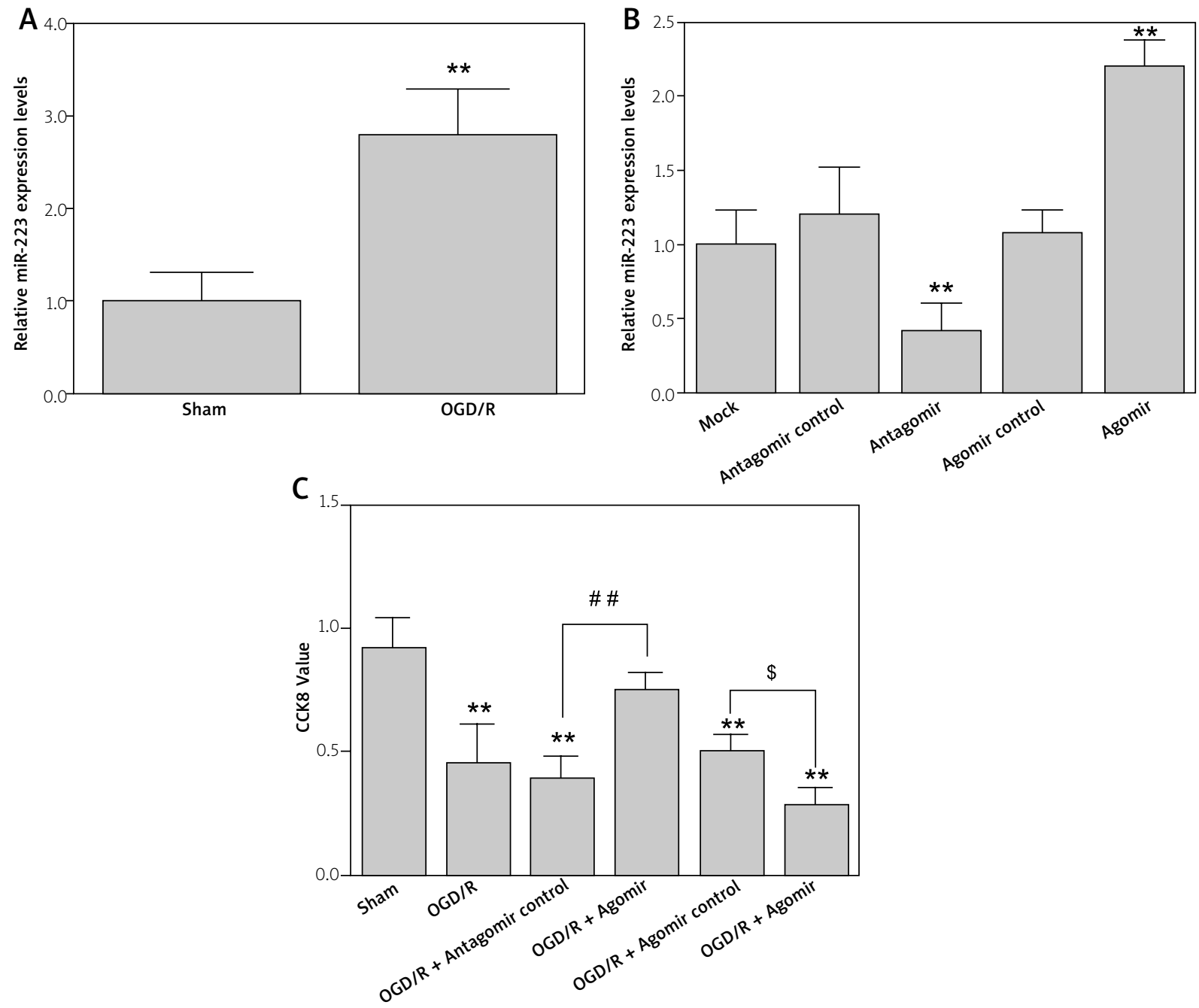

Fig. 1. Up-regulated expression of miR-223 after OGD/R. A) The expression level of miR-223 in cerebral cortex neurons tested by q-PCR after OGD/R treatment. B) The expression level of miR-223 in cerebral cortex neurons treated with miR-223 antagomir and miR-223 agomir. C) Cell proliferation of cerebral cortex neurons subject to $\mathrm{OGD} / \mathrm{R}$ and treated with antagomir, agomir or their corresponding controls tested by CCK 8 assay. $N=5,{ }^{* *} p<0.01,{ }^{\# \# p}<0.01,{ }^{\$} p<0.05$.

that the IGF1R expression level was more significantly decreased in the OGD/R+ antagomir control group than that of the sham group $(p<0.01)$. Furthermore, the level of IGF1R was more significantly increased in the OGD/R+ antagomir than that in OGD/R+ antagomir control group (Fig. 2A) ( $p<0.05$ ). The protein expression level IGF1R was consistent with the mRNA expression level (Fig. 2B-C). These observations indicated that the up-regulating of miR223 may suppress the expression of IGF1R and the down-regulating of miR223 may up-regulate the expression of IGF1R.

\section{The mutual regulatory relationship between miR-223 and IGF1R}

The Targetscan prediction results showed that miR-223 could bind to IGF1R through the binding sites presented in Figure 3A. Luciferase reporter assay was then performed to confirm the interaction between miR-223 and IGF1R. As shown in Figure 3B, the luciferase activity of pmirGLO-WT was significantly suppressed in the miR223-agomir group ( $p<0.01$ ). No significant change was observed in the luciferase activity of pmirGLO-Mut between the miR223-agomir control and miR223-agomir group. These observa- 

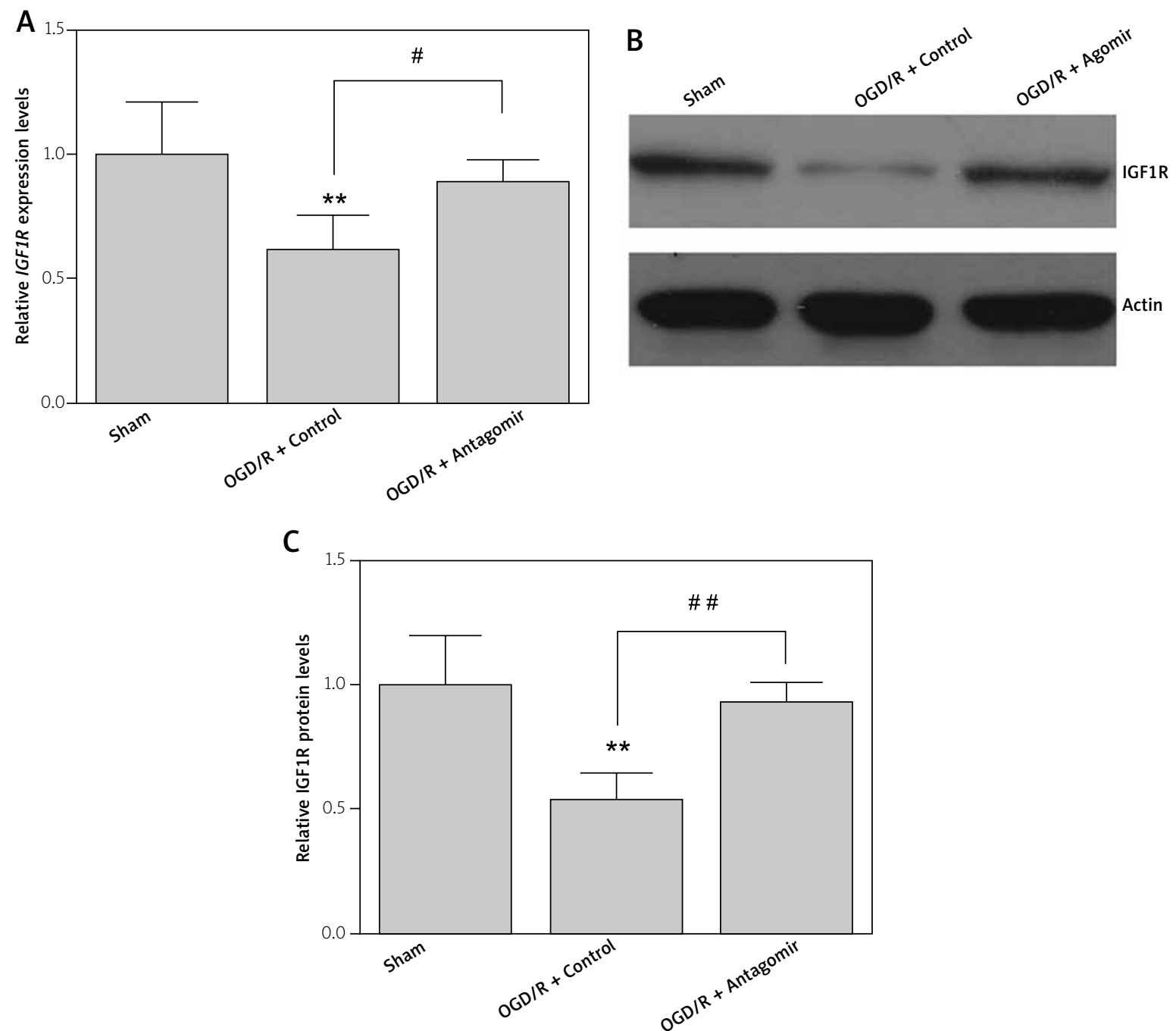

Fig. 2. The influence of miR-223 on IGF1R expression. A) The expression level of IGF1R in cerebral cortex neurons tested by q-PCR after OGD/R or OGD/R combined with antagomir treatment. B) Analysis of IGF1R protein expression in $\mathrm{OGD} / \mathrm{R}$ or $\mathrm{OGD} / \mathrm{R}$ combined with antagomir-treated cerebral cortex neurons via western blot. C) Quantification of IGF1R protein expression levels. $N=5,{ }^{* *} p<0.01,{ }^{\#} p<0.05,{ }^{\# \#} p<0.01$.

tions demonstrated that miR-223 can bind to IGF1R and may be involved in the ischemic brain injury by regulating the expression of IGF1R.

\section{miR-223 participates in OGD/R by regulating the expression of IGF1R}

In order to further investigate the regulatory relationship between miR-223 and IGF1R, siRNA1 IGF1R, SiRNA2 IGF1R, siRNA control, vector or pcDNA-TGF1R was transfected into cortical neurons. The expression level of IGF1R in the siRNA1 and siRNA2 group was dramatically decreased and its level was significant- ly increased in the IGF1R group (Fig. 4A) $(p<0.01)$. The protein level of IGF1R in each group was consistent with the IGF1R mRNA expression level in the corresponding group (Fig. 4B-C). Through CCK8 assay, we found that the $\mathrm{OD}_{450}$ value was significantly decreased in the control +siRNA1 group $(p<0.05)$, control +siRNA2 group $(p<0.01)$ and agomir + vector group $(p<0.01)$ compared with the mock group. However, $\mathrm{OD}_{450}$ value was dramatically increased in the control + IFG1R group $(p<0.05)$ and antagomir + control group $(p<0.01)$ compared with the mock group. In antagomir + siRNA1 and antagomir + siRNA2 
A

WT IGF1R: $\quad$ 5'CCUACCCAAACCCUUAACUGACA 3'
mmR-223:

Mut IGF1R: $\quad$ CCUACCCAAACCCUUUCAAUUUU 3'

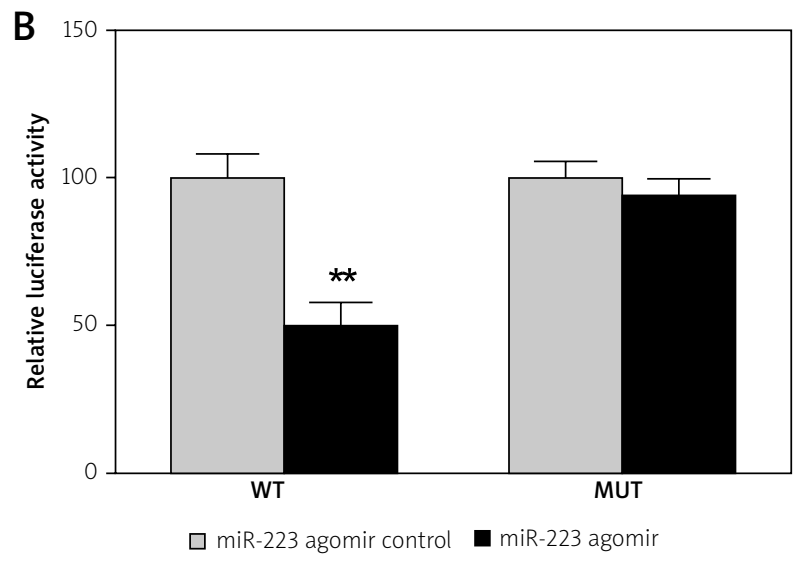

Fig. 3. The mutual regulatory relationship between miR-223 and IGF1R. A) The predicted binding sites between miR-223 and IGF1R. B) The luciferase activity of pmirGLO-WT and pmirGLO-Mut tested by luciferase reporter assay. $N=5,{ }^{* *} p<0.01$.

groups, $\mathrm{OD}_{450}$ value was obviously lower than in the antagomir + control group. Furthermore, $\mathrm{OD}_{450}$ value in the agomir + vector group was significantly lower than in the antagomir + IGF1R group. These observations indicated that miR-223 participates in OGD/R by regulating the expression of IGF1R.

\section{Discussion}

Previous studies have indicated that miR-223 can serve as potential biomarkers for gastric cancer [14] and sepsis [21]. Furthermore, the up-regulation of miR-223 has been demonstrated in mice with hepatic ischemia/reperfusion injury [27], rat transient MCAO [2], ischemic preconditioning [13], the blood of acute ischemic stroke patients [23] and young stroke patients [20]. However, the specific mechanism of miR-223 in cerebral ischemia is still unclear. Here, we have constructed an OGD/R model of cortical neurons and found that the level of miR-223 was increased similarly in cortical neurons treated with OGD/R. Furthermore, overexpression of miR-223 enhanced the inhibitory effect on cell proliferation, whereas it down-regulated the miR-223 level providing protective effects against the $O G D / R$ injury (Fig. 1). Previously, it was reported that overexpression of miR-223 also caused suppression on HeLa cell proliferation [24]. The inhibitory effect of miR-223 on cell growth that we observed in cortical neurons treated with OGD/R was consistent with its role in hepatocellular carcinoma. These data further illustrate that miR-223 plays a role in cerebral ischemia.
In order to explore the potential mechanism and find out the target mRNA of miR223 which may be involved in the ischemic brain injury, the targetscan algorithm was used. We found that the expression of IGF1R was negatively correlated with miR-223 expression and was a potential target of miR-223. It had been universally understood that IGF1R was up-regulated in many kinds of cancers and serves as an oncogene [7]. Researchers have demonstrated that IGF1R was the target of miR-143, miR-99a and miR-133a [4]. However, the relationship between miR-223 and IGF1R was still controversial. In HeLa, leukemia and hepatoma cells, IGF1R had been demonstrated to be the target of miR-223 [9], while no obvious correlation between miR-223 and IGF1R was found in the blood of acute ischemia patients [22]. To explore whether miR-223 could directly regulate IGF1R expression, q-PCR, western blot and Luciferase reporter assays were performed. We observed that both the mRNA and protein level of IGF1R were decreased when cortical neurons were treated with $\mathrm{OGD} / \mathrm{R}$, while the IGF1R levels were increased when miR-223 antagomir was added (Fig. 2). Furthermore, an obvious decline in luciferase activity was observed when miR-223 was over-expressed. When the binding sites between miR223 and IGF1R were mutated on IGF-1R 3'UTR, the interaction between them disappeared (Fig. 3). These phenomena strongly suggested that IGF1R interacted with miR223 and was inhibited at both transcriptional and translational levels. Our experimental results showing that IGF1R was a target of miR223 are consistent with the results obtained in HeLa, leukemia 

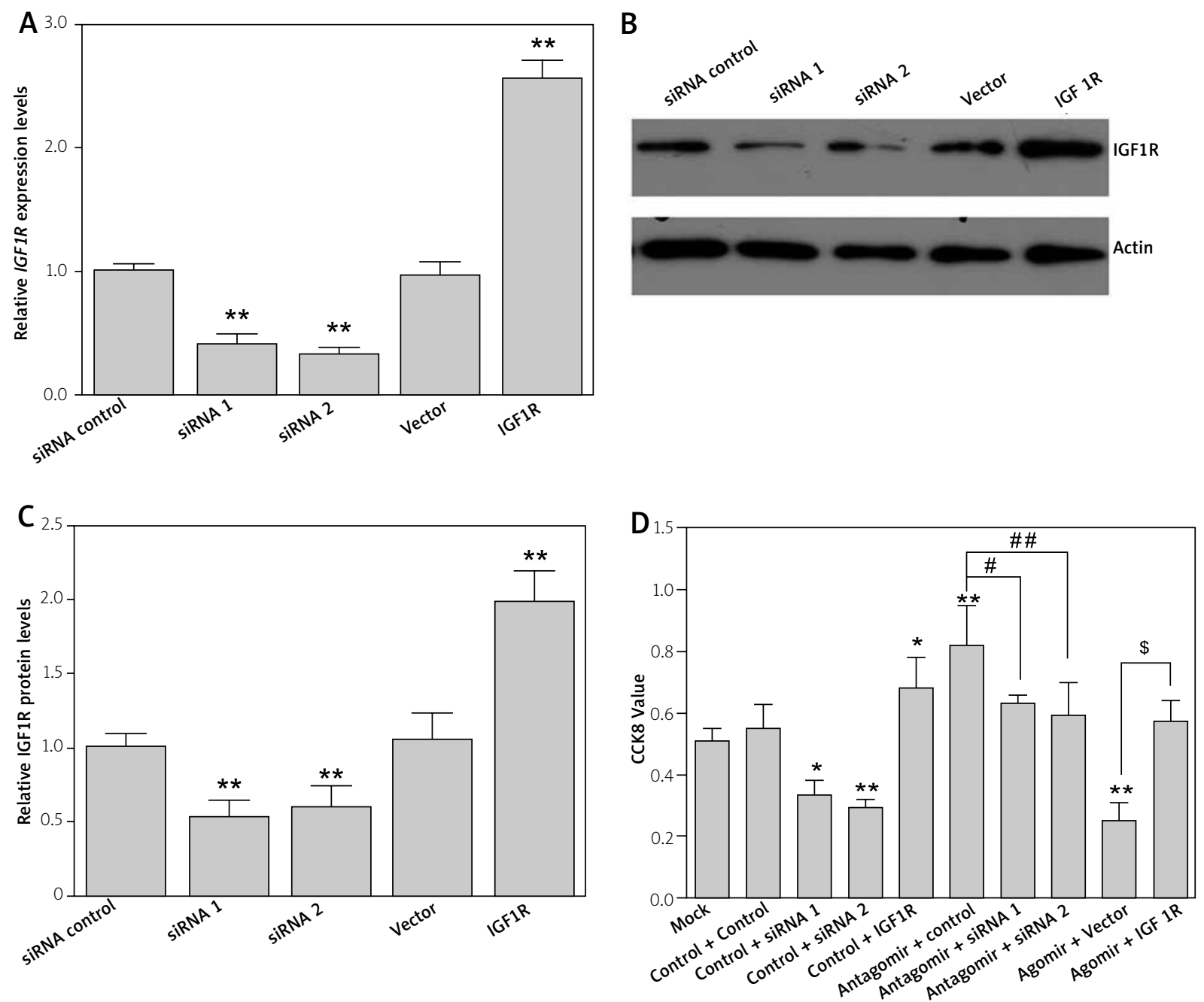

Fig. 4. miR-223 participates in OGD/R by regulating the expression of IGF1R. A) q-PCR analysis of IGF1R expression in cortical neurons transfected with siRNA1 IGF1R, siRNA2 IGF1R, siRNA control, vector or pcDNATGF1R. B) Western blot analysis of the IGF1R protein level in cortical neurons transfected with siRNA1 IGF1R, SiRNA2 IGF1R, siRNA control, vector or pcDNA-TGF1R. C) Quantification of IGF1R protein expression levels in (B). D) CCK8 assay was performed to detect neuron proliferation in cortical neurons. $N=5,{ }^{\star} p<0.05,{ }^{* *} p<0.01$, ${ }^{\#} p<0.05,{ }^{\#} p<0.01,{ }^{\$} p<0.05$.

and hepatoma cells in vitro [9]. These data further confirmed that miR223 could regulate the expression of IGF1R. While, no significance correlation between miR-223 and IGF1R was found from the acute ischemic stroke patients in vivo [22]. These inconsistencies may be caused by the differences between the in vivo and in vitro environments. Furthermore, cortical neurons from Sprague-Dawley rats were used in our study, while blood samples from acute ischemic stroke patients were used in the study of Wang et al. [22]; this may be another reason for this difference.
To further demonstrate whether miR-223 participates in OGD/R by regulating the expression of IGF1R, we transfected silGF1R into cortical neurons. The expression level of IGF1R was significantly decreased after silGF1R transfection and resulted in a similar inhibition of cell viability to the suppression caused by miR-223 (Fig. 4). On the other hand, we found that over-expressed IGF1R in the miR-223 agomir group can abolish the suppression of miR-223. These phenomena demonstrated that miR-223 suppressed proliferation of cortical neurons treated with OGD/R 
via inhibiting IGF1R expression. Whereas, Harraz et al. have found that miR-223 played a neuroprotective role in hippocampal neurons by regulating the expression and function of GluR2 and NR2B subunits of the glutamate receptor [16]. The contradiction between our results and theirs indicated that miR-223 overexpression may promote ischemia-induced cell death or play a neuroprotective role in stroke depending on the areas of the brain [16].

In summary, this research reported that miR-223 was up-regulated in OGD/R-treated cortical neurons and it showed an inhibitory effect on cortical neurons cell proliferation. Furthermore, our results indicated that IGF1R might be an important target of miR-223 and the expression level of IGF1R was negatively associated with the level of miR-223. These results indicated that miR-223 might play an important role in ischemic brain injury and suppress proliferation of cortical neurons via inhibiting IGF1R expression. The specific relationship between miR223 and IGF1R in ischemic brain injury needs more in vivo evidence to support.

\section{Disclosure}

The authors report no conflict of interest.

\section{References}

1. Blakeley JO, Llinas RH. Thrombolytic therapy for acute ischemic stroke. J Neurol Sci 2007; 261: 55-62.

2. Dharap A, Bowen K, Place R, Li LC,Vemuganti R. Transient focal ischemia induces extensive temporal changes in rat cerebral microRNAome. J Cereb Blood Flow Metab 2009; 29: 675-687.

3. Di Y, Lei Y, Yu F, Changfeng F, Song W, Xuming M. MicroRNAs expression and function in cerebral ischemia reperfusion injury. J Mol Neurosci 2014; 53: 242-250.

4. Gong Y, Ren J, Liu K, Tang LM. Tumor suppressor role of miR-133a in gastric cancer by repressing IGF1R. World J Gastroenterol 2015; 21: 2949-2958.

5. Gottardo F, Liu CG, Ferracin M, Calin GA, Fassan M, Bassi P, Sevignani C, Byrne D, Negrini M, Pagano F, Gomella LG, Croce CM, Baffa R. Micro-RNA profiling in kidney and bladder cancers. Urol Oncol 2007; 25: 387-392.

6. Haneklaus M, Gerlic M, O'Neill LA, Masters SL. miR-223: infection, inflammation and cancer. J Intern Med 2013; 274: 215-226.

7. Hartog H, Wesseling J, Boezen HM, van der Graaf WT. The insulin-like growth factor 1 receptor in cancer: old focus, new future. Eur J Cancer 2007; 43: 1895-1904.

8. Huang W, Liu X, Cao J, Meng F, Li M, Chen B, Zhang J. miR-134 regulates ischemia/reperfusion injury-induced neuronal cell death by regulating CREB signaling. J Mol Neurosci 2015; 55: 821-829.
9. Jia CY, Li HH, Zhu XC, Dong YW, Fu D, Zhao QL, Wu W, Wu XZ. MiR-223 suppresses cell proliferation by targeting IGF-1R. PLoS One 2011; 6: e27008.

10. Jiang LH, Yuan XL, Yang NY, Ren L, Zhao FM, Luo BX, Bian YY, Xu JY, Lu DX, Zheng YY, Zhang CJ, Diao YM, Xia BM, Chen G. Daucosterol protects neurons against oxygen-glucose deprivation/reperfusion-mediated injury by activating IGF1 signaling pathway. J Steroid Biochem Mol Biol 2015; 152: 45-52.

11. Khan AA, Betel D, Miller ML, Sander C, Leslie CS, Marks DS. Transfection of small RNAs globally perturbs gene regulation by endogenous microRNAs. Nat Biotechnol 2009; 27: 549-555.

12. Laios A, O'Toole S, Flavin R, Martin C, Kelly L, Ring M, Finn SP, Barrett C, Loda M, Gleeson N, D’Arcy T, McGuinness E, Sheils O, Sheppard B, J OL. Potential role of miR-9 and miR-223 in recurrent ovarian cancer. Mol Cancer 2008; 7: 35.

13. Lee ST, Chu K, Jung KH, Yoon HJ, Jeon D, Kang KM, Park KH, Bae EK, Kim M, Lee SK, Roh JK. MicroRNAs induced during ischemic preconditioning. Stroke 2010; 41: 1646-1651.

14. Li BS, Zhao YL, Guo G, Li W, Zhu ED, Luo X, Mao XH, Zou QM, Yu PW, Zuo QF, Li N, Tang B, Liu KY, Xiao B. Plasma microRNAs, miR-223, miR-21 and miR-218, as novel potential biomarkers for gastric cancer detection. PLoS One 2012; 7: e41629.

15. Lin D, Li G, Zuo Z. Volatile anesthetic post-treatment induces protection via inhibition of glycogen synthase kinase $3 \beta$ in human neuron-like cells. Neuroscience 2011; 179: 73.

16. Harraz MM, Eacker SM, Wang X, Dawson TM, Dawson VL. MicroRNA-223 is neuroprotective by targeting glutamate receptors. P Natl Acad Sci USA 2012; 109: 18962-18967.

17. Mozaffarian D, Benjamin EJ, Go AS, Arnett DK, Blaha MJ, Cushman M, De FS, Després JP, Fullerton HJ, Howard VJ. Heart disease and stroke statistics--2015 update: a report from the American Heart Association. Circulation 2012; 125: e2.

18. Riedemann J, Macaulay VM. IGF1R signalling and its inhibition. Endocr Relat Cancer 2006; 13 Suppl 1: S33-43.

19. Stumm R, Kolodziej A, Prinz V, Endres M, Wu DF, Höllt V. Pituitary adenylate cyclase-activating polypeptide is up-regulated in cortical pyramidal cells after focal ischemia and protects neurons from mild hypoxic/ischemic damage. I Neurochem 2007; 103: 1666-1681.

20. Tan KS, Armugam A, Sepramaniam S, Lim KY, Setyowati KD, Wang CW, Jeyaseelan K. Expression profile of MicroRNAs in young stroke patients. PLoS One 2009; 4: e7689.

21. Wang JF, Yu ML, Yu G, Bian JJ, Deng XM, Wan XJ, Zhu KM. Serum miR-146a and miR-223 as potential new biomarkers for sepsis. Biochem Bioph Res Co 2010; 394: 184.

22. Wang Y, Zhang Y, Huang J, Chen X, Gu X, Wang Y, Zeng L, Yang GY. Increase of circulating miR-223 and insulin-like growth factor- 1 is associated with the pathogenesis of acute ischemic stroke in patients. BMC Neuro 2014; 14: 1-7.

23. Wang Y, Zhang Y, Huang J, Chen X, Gu X, Wang Y, Zeng L, Yang GY. Increase of circulating miR-223 and insulin-like growth factor-1 is associated with the pathogenesis of acute ischemic stroke in patients. BMC Neurol 2014; 14: 77.

24. Wong QW, Lung RW, Law PT, Lai PB, Chan KY, To KF, Wong N. MicroRNA-223 is commonly repressed in hepatocellular carcinoma and potentiates expression of Stathmin1. Gastroenterology 2008; 135: 257-269. 
25. Yan W, Fang Z, Yang Q, Dong H, Lu Y, Lei C, Xiong L. SirT1 mediates hyperbaric oxygen preconditioning-induced ischemic tolerance in rat brain. J Cereb Blood Flow Metab 2013; 33: 396.

26. Yang L, Xiong Y, Hu XF, Du YH. MicroRNA-323 regulates ischemia/reperfusion injury-induced neuronal cell death by targeting BRI3. Int J Clin Exp Pathol 2015; 8: 10725-10733.

27. Yu CH, Xu CF, Li YM. Association of MicroRNA-223 expression with hepatic ischemia/reperfusion injury in mice. Dig Dis Sci 2009; 54: 2362-2366.

28. Yuan Y, Zhang X, Zheng Y, Chen Z. Regulation of mitophagy in ischemic brain injury. Neurosci Bull 2015; 31: 395-406. 